\title{
Transfer of a chromosomal locus responsible for mucoid colony morphology in Pseudomonas aeruginosa isolated from cystic fibrosis patients to $P$. aeruginosa PAO
}

\author{
J. MACGEORGE, V. KOROLIK, A. F. MORGAN, V. ASCHE* and B. W. HOLLOWAY
}

Department of Genetics, Monash University, Clayton, Victoria 3168, and "Department of Microbiology, Queen Victoria Medical Centre, 172 Lonsdale Street, Melbourne, Victoria 3000, Australia

\begin{abstract}
Summary. The locus responsible for mucoid colony morphology in five independent clinical isolates of Pseudomonas aeruginosa from cystic fibrosis patients have been transferred by means of pM060-mediated conjugation to the genetically characterised strain $P$. aeruginosa PAO. Genetic mapping has shown that in all five strains the locus is on the chromosome between $89^{\prime}$ and $94^{\prime}$, although it is not possible to say that the same locus is involved in each case. The way is now open for a more detailed genetic analysis of the loci responsible for mucoid colony morphology.
\end{abstract}

\section{Introduction}

The lungs of patients suffering from cystic fibrosis (CF) invariably become infected with Pseudomonas aeruginosa (May et al., 1972). Antibiotic treatment may not eradicate the organism but is usually clinically beneficial (Hodson et al., 1981). Inevitably, however, a $P$. aeruginosa variant known as "mucoid" (from the appearance of the colonies upon culture) appears in the lung (Doggett et al., 1966), and subsequently the prognosis is poor (Wood et al., 1976; Henry et al., 1982).

The mucoid colonial characteristics of such $P$. aeruginosa variants are due to the production of alginic acid, a 1,4-linked, selectively acetylated, copolymer of $\beta$-D-mannuronic acid and its $\mathrm{C}-5$ epimer $\alpha$-L-glucuronic acid (Fyfe and Govan, 1983). The enzymology of alginic acid synthesis has not yet been elucidated. Pugashetti et al. (1983) suggested that it is synthesised via the Entner-Doudoroff pathway - G-P-D mannose dehydrogenase, which converts G-P-D mannose to G-P-D mannuronate, being the major enzyme-whereas Banerjee et al. (1983) postulated that synthesis occurs via glyceraldehyde-3-phosphate.

Two different approaches have been used in attempts to elucidate the genetics of mucoid variation. Established systems of gene transfer and recombination are a basic prerequisite of any

Received 7 May 1985; accepted 31 Jul. 1985. genetic study, and $P$. aeruginosa strain PAO is the most extensively characterised pseudomonad in this respect (Holloway et al., 1979; Holloway and Matsumoto, 1984). In the first approach Govan and Fyfe (1978) and Fyfe and Govan (1983) have studied the genetics of mucoid mutants derived from $P$. aeruginosa PAO. However, there is no assurance that such mutants bear any relationship to mucoid strains of $P$. aeruginosa isolated from $\mathrm{CF}$ patients.

The second approach was to take a clinical mucoid strain of $P$. aeruginosa, introduce into it auxotrophic and non-mucoid mutations, and by use of chromosome-mobilising plasmids previously described for PAO, attempt to map the loci involved in mucoid production in that strain (Ohman and Chakrabarty, 1981). A difficulty of this approach is that there is no easy way of relating auxotrophic and other loci mapped in this manner to the established $P$. aeruginosa PAO genetic map.

We have used a third approach which minimises objections to the above methods. We have performed experiments to demonstrate the conjugal transfer of chromosomal loci (muc) causing the mucoid phenotype to $P$. aeruginosa $\mathrm{PAO}$, and to relate these loci to markers on the $P$. aeruginosa PAO genetic map. In these crosses we have used the enhanced chromosome mobilising (ECM) plasmid pM060 (Riess et al., 1980), which is similar to R68.45 (Haas and Holloway, 1976) but showed much greater stability of chromosome-mobilising ability. 


\section{Materials and methods}

\section{Bacterial strains and plasmids}

These are listed in table I.

\section{Media and cultural conditions}

Nutrient Yeast Broth, Nutrient Agar, Minimal Agar (all described by Stanisich and Holloway, 1972) and
Medium P (Leisinger et al., 1972) were used for the culture of $P$. aeruginosa PAO. For mucoid clinical isolates and mucoid recombinant (PAM) strains, fructose peptone broth (Difco Bacto-peptone 1\% w/v, fructose 0.3\% $\mathrm{w} / \mathrm{v}$ in distilled water) and peptone fructose agar (fructose peptone broth solidified with Difco Bacto-agar $1.5 \% \mathrm{w} / \mathrm{v}$ ) were used. Stocks of amino acids and purines $(50 \mathrm{mM})$ were stored over chloroform and used at a final concentration of $1 \mathrm{~mm}$ except for isoleucine which was used

Table I. Bacterial strains and plasmids

\begin{tabular}{|c|c|c|}
\hline Strain & Genotype or phenotype & Derivation or reference \\
\hline \multicolumn{3}{|l|}{ Bacterium } \\
\hline Ps3694 & Prototroph, $\mathrm{Muc}^{+}$ & This study \\
\hline Ps3694-2 & met-3003, str-3001, $\mathrm{Muc}^{+}$ & Met - Str $^{t}$ derivative of Ps3694 \\
\hline Ps 3710 & Prototroph, $\mathrm{Muc}^{+}$ & This study \\
\hline Ps $3710-17$ & trp-3002, $\mathrm{Muc}^{+}$ & $\operatorname{Trp}^{-}$derivative of Ps3710 \\
\hline Ps3712 & Prototroph, $\mathrm{Muc}^{+}$ & This study \\
\hline Ps3712-15 & leu-3002, Muc ${ }^{+}$ & Leu $^{-}$derivative of Ps3712 \\
\hline Ps3738 & Prototroph, $\mathrm{Muc}^{+}$ & This study \\
\hline Ps3738-1 & lys $-3002, \mathrm{Muc}^{+}$ & Lys- derivative of Ps3738 \\
\hline Ps3739 & Prototroph, $\mathrm{Muc}^{+}$ & This study \\
\hline Ps3739-1 & leu-3004, Muc ${ }^{+}$ & Leu $^{-}$derivative of Ps3739 \\
\hline PAM207 & thr-9001, nal-26, muc-3712 & $\begin{array}{l}\text { Pur }^{+} \mathrm{Cys}^{+} \text {Pro }^{+} \mathrm{Muc}^{+} \\
\text {recombinant from the cross } \\
\text { PS3712-15(pM060) } \times \text { PAO1052 }\end{array}$ \\
\hline PAM301 & $l y s-12$, muc-3694 & $\begin{array}{l}\mathrm{Cys}^{+} \mathrm{His}^{+} \mathrm{Muc}^{+} \\
\text {recombinant from the cross } \\
\text { Ps3694(pMO60) } \times \text { PAO542 }\end{array}$ \\
\hline PAM404 & $l y s-12, m u c-3739$ & $\begin{array}{l}\mathrm{Cys}^{+} \mathrm{His}^{+} \mathrm{Muc}^{+} \\
\text {recombinant from the cross } \\
\text { Ps3739-1(pMO60) } \times \text { PAO542 }\end{array}$ \\
\hline PAM501 & $\arg A 171$, nal -25, muc- -3738 & $\begin{array}{l}\mathrm{Cys}^{+} \mathrm{His}^{+} \mathrm{Pro}^{+} \mathrm{Muc}^{+} \\
\text {recombinant from the cross } \\
\text { Ps3738-1(pMO60) } \times \text { PAO2022 }\end{array}$ \\
\hline PAM601 & $l y s-12, m u c-3710$ & $\begin{array}{l}\mathrm{Cys}^{+} \mathrm{His}^{+} \mathrm{Muc}^{+} \\
\text {recombinant from the cross } \\
\text { Ps3710-17(pMO60) } \times \text { PAO542 }\end{array}$ \\
\hline PAO542 & cys -5606, his -5075, lys -12 & Royle et al. $(1981)^{*}$ \\
\hline PAO1052 & $\begin{array}{l}\text { pur-67, thr-9001, cys-59 } \\
\text { proB } 65, \text { nal }-26\end{array}$ & Royle et al. (1981)* \\
\hline $\mathrm{PAO} 2022$ & $\begin{array}{l}\text { cys-5606, his-5075, arg A171 } \\
\text { pro-67, nal-25 }\end{array}$ & Fyfe and Govan (1980)* \\
\hline PAO6020 & pur -67, cys -59, proB 65 & Soldati et al. (1984)* \\
\hline $\begin{array}{l}\text { PAO6021 } \\
\text { Plasmid }\end{array}$ & proB65, pru-365 & Soldati et al. (1984)* \\
\hline pMO60 & $\mathrm{Cb} \mathrm{Km} / \mathrm{Nm}$ Tc Tra Cma & Riess et al. (1980) \\
\hline pMO887 & $\mathrm{Cb} \mathrm{Km} / \mathrm{Nm} \mathrm{Tc}{ }^{\mathrm{s}}$ Tra Cma & M. Nayudu $\dagger$ \\
\hline pKT212 & $\mathrm{Sm} \mathrm{Tc} \mathrm{Cm}$ & Bagdasarian et al. (1979) \\
\hline
\end{tabular}

$P$. aeruginosa PAO strains are derived from PAOl (ATCC 15692), Ps strains are clinical isolates of $P$. aeruginosa and PAM strains are recombinants derived from conjugal crosses between mucoid clinical isolates and PAO which exhibit the mucoid phenotype. Genotype symbols are the same as those used by Bachmann (1983) for Escherichia coli except that str signifies streptomycin resistance, nal resistance to nalidixic acid, pru proline utilisation and muc involvement in mucoid colony phenotype. Phenotype symbols: $\mathrm{Cb}$, carbenicillin resistance, $\mathrm{Km} / \mathrm{Nm}$, kanamycin/ neomycin resistance; Tc, tetracycline resistance; $\mathrm{Sm}$, streptomycin resistance; $\mathrm{Cm}$, chloramphenicol resistance; Tra, conjugative ability; Cma, chromosome mobilizing ability; Muc, mucoid colony morphology (Muc ${ }^{+}$, mucoid; $\mathrm{Muc}^{-}$, nonmucoid).

*The map positions of markers relevant to this study are shown in the figure.

$\uparrow$ pMO887 is a Tc ${ }^{\mathrm{s}}$ derivative of R68.45 (Haas and Holloway, 1976), and carries a small deletion, generated in vitro, from the SalI site adjacent to the Tc gene (M. Nayudu, personal communication). 
at $0.5 \mathrm{~mm}$. When appropriate, media were supplemented with (/L) carbenicillin (Pyopen; Beecham, Australia) 500 $\mathrm{mg}$, streptomycin (Sigma Chemical Co.) $500 \mathrm{mg}$, or tetracycline (oxytetracycline tetrachloride; Sigma) 200 mg. Strains with ability to use proline as sole carbon source $\left(\mathrm{Pru}^{+}\right)$were selected on Medium $\mathrm{P}$ supplemented with proline $0 \cdot 1 \% \mathrm{w} / \mathrm{v}$. All cultures were incubated at $37^{\circ} \mathrm{C}$, except PAO strains to be used as recipients in crosses with clinical strains which were grown in nutrient yeast broth at $43^{\circ} \mathrm{C}$; this renders $P$. aeruginosa PAO phenotypically restrictionless for about 60 generations of growth at $37^{\circ} \mathrm{C}$ (Holloway, 1965).

\section{Bacteriological techniques}

Plate matings (Stanisich and Holloway, 1972) and carbenicillin semi-selection of auxotrophs after mutagenesis with ethyl methane sulphonate (Sigma) (Watson and Holloway, 1976) were performed as described. Pyocin typing of clinical isolates was performed by the method of Gillies and Govan (1966).

\section{Results}

During a 2-year period we have received more than 150 mucoid clinical isolates of $P$. aeruginosa from CF patients at the Royal Children's Hospital, Melbourne. These isolates showed considerable variation in growth rate and in the amount of alginate produced on peptone fructose agar; interestingly, in view of the often reported instability of the mucoid phenotype upon subculture, most of our strains were quite stable (data not shown). We chose for further study five stable mucoid isolates (listed in table I) that showed a good growth rate and which had been isolated from unrelated patients. Of these five isolates, only Ps3738 could be distinguished from the rest by pyocin typing (Gillies and Govan, 1966); it was of pyocin type 29, the other four being of type 5. Of the four type- 5 isolates, Ps3694 and Ps3710 were gentamicin resistant, whereas Ps3712 and Ps3739 were gentamicin sensitive (information provided by the Royal Children's Hospital). Thus it is not possible for us to state unequivocally that our five isolates represent more than three genetically distinct strains.

\section{Transfer of muc loci to $P$. aeruginosa $P A O$}

Initial attempts to transfer the gene or genes responsible for the mucoid phenotype to $P$. aeruginosa PAO involved the use of the ECM plasmid pMO887 which does not carry genes for tetracycline resistance $\left(\mathrm{Tc}^{\mathrm{R}}\right)$. This plasmid was transferred to a mucoid clinical isolate by selection for carbenicillin resistance. This strain was then used as a donor with various multi-marked PAO recipients carrying the IncQ plasmid pKT212, which confers $T c^{R}$, with selection for both $\mathrm{Tc}^{\mathrm{R}}$ and prototropy of individual auxotrophic markers. The rationale behind this approach is that we have never observed mutation to high-level tetracycline resistance in $P$. aeruginosa. A similar procedure was used successfully to allow large numbers of clinical isolates of $P$. aeruginosa to be readily screened for chromosome-mobilising plasmids (Dean et al., 1979). However, for the analysis of mucoid strains this approach proved to be unsatisfactory for two reasons. First, although pMO887 is stable in $P$. aeruginosa PAO it was highly unstable in all five clinical isolates, losing chromosome-mobilising ability at a high frequency. Second, a more serious problem was "back transfer" of pKT212 to the clinical isolate; pKT212 was mobilised by pMO887 which had transferred to the PAO recipient at high frequency without direct selection. This transfer of pKT212 resulted in the mucoid donors acquiring $\mathrm{Tc}^{\mathrm{R}}$ and growing on the mating plates.

These problems were overcome by introducing ethyl methane sulphonate-induced auxotrophic mutations into each of the five clinical isolates to provide a contraselective marker, and by transferring pMO60 to each clinical strain by selection for carbenicillin resistance. This naturally occurring ECM plasmid proved to be quite stable in all five clinical isolates tested. Auxotrophic derivatives of the clinical isolates, carrying pMO60, were plate mated with various multi-marked $P$. aeruginosa PAO recipients, selection was applied for protrophy of single markers, and the mating plates were screened for the appearance of mucoid recombinants. For each clinical isolate donor, prototrophic

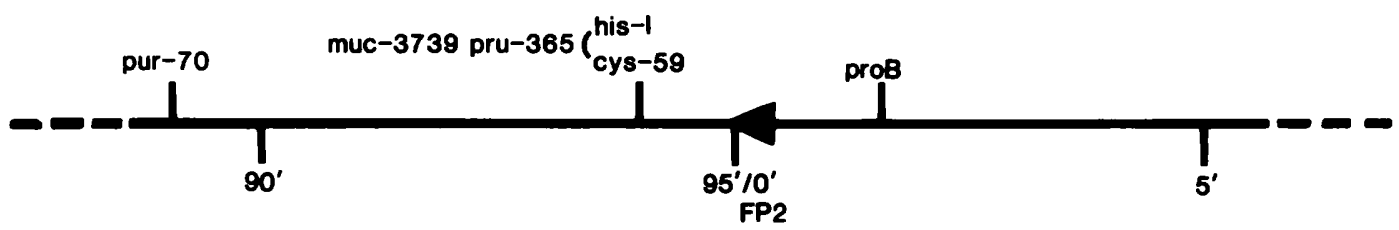

Figure. Genetic map of the FP2 origin region of P. aeruginosa PAO, taken from Holloway and Matsumoto (1984); pro-65 and pro-67 are alleles of the proB locus, pur-67 is closely linked to pur-70, cys-5606 is closely linked to cys -59 , and his-5075 is an allele of the hisI locus (Royle et al., 1981). Markers used for contraselection in plate matings were: $\arg A 171\left(18^{\prime}\right)$, lys $-12\left(20^{\prime}\right)$ and $t h r-9001\left(43^{\prime}\right)$ (Holloway and Matsumoto, 1984). 
recombinants were formed at a frequency of about $10^{-6} /$ donor cell, which is about $10-100$-fold less than would be expected in PAO $\times$ PAO (pMO60) plate matings (data not shown). When selection was made for prototrophy of markers in the pur-70pro $B$ region of the chromosome (see figure), $10-15 \%$ of the recombinant colonies were mucoid when Ps3694-2, Ps3710-17 or Ps3712-15 carrying pMO60 were the donors, but with Ps3738-1 and Ps3739-1 carrying pMO60 as donors, only about $1 \%$ of the recombinant colonies were mucoid (data not shown). With all five clinical isolates, however, selection for other markers failed to yield any mucoid recombinants (data not shown). This result suggested that the muc gene or genes being transferred had a chromosomal location; if a plasmid was being mobilised from the clinical isolates apparent linkage to all chromosomal markers used to select recombinants would be expected. It was assumed that the mucoid PAO derivatives now carried a small region (probably $5^{\prime}-10^{\prime}$, and including the $m u c$ gene or genes) of chromosome from the clinical isolates in place of the homologous PAO chromosomal region.

\section{Mapping of the muc locus}

To determine the location of the muc gene or genes more accurately, one recombinant mucoid clone from each cross was chosen for further study. We have designated these five clones as PAM strains, and the particular derivation of each is shown in table I. Selection for carbenicillin resis- tance was used to transfer pMO60 into each of the PAM strains which were then used as donors in plate matings with strain PAO1052, or strain PAO6020 in the case of PAM207 (pMO60) because PAM207 required threonine. Subsequent selection was made for one or more prototrophic marker. The co-inheritance values obtained from the cross PAM404 (pMO60) $\times$ PAO1052 are shown in table II. As expected, $m u c$ is linked to all three markers in the pur-70-proB region but unlinked to $t h r-48$ (figure). When $c y s-59^{+}$was the selected marker the observation that none of the $\mathrm{Muc}^{-}$recombinants were $\mathrm{Pur}^{+}$, whereas $88 \%$ of them were Pro ${ }^{+}$ suggested that muc is to the left of cys-59 in the figure. This was confirmed when cys $-59^{+}$and pur$70^{+}$were selected simultaneously because all of the $\mathrm{Cys}^{+} \mathrm{Pur}^{+}$recombinants were $\mathrm{Muc}^{+}$, which indicates that muc is located between pur-70 and cys59. Although it would be possible to obtain such a result if muc were located to the left of pur-70 providing that they were very lightly linked, it was found that the co-inheritance of pur-70 and $m u c$ 3739 was only $37 \%$.

Matings between the other four PAM strains carrying pMO60 and PAO1052, or PAO6020 in the case of PAM207 (pMO60), gave similar results. Thus all five PAM strains behaved as though they differed from PAO at a single muc locus, located between pur-70 and cys-59. However, for a given PAM strain it is not possible to say that only one muc locus is involved in producing the mucoid phenotype. Although the $\mathrm{Muc}^{+}$recombinant colonies from a given PAM $(\mathrm{pMO} 60) \times$ PAO cross

Table II. Conjugational mapping of the muc-3739 mutation in P. aeruginosa PAO

\begin{tabular}{|c|c|c|c|c|c|c|}
\hline \multirow{2}{*}{$\begin{array}{l}\text { Selected } \\
\text { marker(s) }\end{array}$} & \multirow{2}{*}{$\begin{array}{l}\text { Recombination } \\
\text { frequency per } \\
\text { donor cell }\end{array}$} & \multirow{2}{*}{$\begin{array}{l}\text { Mucoid (percentage } \\
\text { of total phenotype } \\
\text { recombinants) }\end{array}$} & \multicolumn{4}{|c|}{$\begin{array}{l}\text { Percentage co-inheritance of } \\
\text { unselected marker(s) }\end{array}$} \\
\hline & & & pur-70+ & cys $-59^{+}$ & proB65+ & $t h r-48^{+}$ \\
\hline \multirow[t]{2}{*}{ pur-70+ } & $9.0 \times 10^{-7}$ & $+(37)$ & $\ldots$ & 90 & 84 & 0 \\
\hline & & $-\quad(63)$ & & 0 & 0 & 0 \\
\hline \multirow[t]{2}{*}{ cys $-59^{+}$} & $2.0 \times 10^{-5}$ & $+(27)$ & 54 & $\ldots$ & 100 & 0 \\
\hline & & $-\quad(73)$ & 0 & $\ldots$ & 88 & 0 \\
\hline \multirow[t]{2}{*}{ proB65+ } & $2 \cdot 2 \times 10^{-5}$ & $+(43)$ & 78 & 100 & $\ldots$ & 0 \\
\hline & & $-(57)$ & 0 & 85 & $\ldots$ & 0 \\
\hline \multirow[t]{2}{*}{$t h r-48^{+}$} & $2.4 \times 10^{-5}$ & $+\quad(0)$ & $\ldots$ & $\ldots$ & $\ldots$ & $\ldots$ \\
\hline & & $-(100)$ & 0 & 0 & 0 & $\ldots$ \\
\hline \multirow[t]{2}{*}{ cys $-59^{+}$pur $-70^{+}$} & $2.9 \times 10^{-7}$ & $+(100)$ & $\ldots$ & $\ldots$ & 70 & 0 \\
\hline & & $-\quad(0)$ & $\ldots$ & $\ldots$ & $\ldots$ & $\ldots$ \\
\hline \multirow[t]{2}{*}{ cys $-59^{+}$proB65+ } & $1.9 \times 10^{-5}$ & $+(77)$ & 81 & $\ldots$ & $\ldots$ & 0 \\
\hline & & - (23) & 0 & $\ldots$ & $\ldots$ & 0 \\
\hline
\end{tabular}

PAM404 (pMO60) was plate mated with PAO1052 and selection made for prototrophy of individual and pairs of markers. In each case one hundred recombinants were scored for co-inheritance of unselected donor markers. The map position of markers is shown in the figure.

$+=$ Mucoid; $-=$ non-mucoid; . . . = not isolated. 
were morphologically indistinguishable, and segregation of $m u c$ loci in these crosses might have been expected to yield mucoid colonies of distinguishable phenotype, it is possible that segregation of muc loci was occurring undetected. However, if more than one locus is involved, such loci must be tightly linked.

It is also impossible to say that the five PAM strains differ from strain PAO at the same locus. Although they were indistinguishable from each other in colony morphology, further genetic analysis is required to answer this point.

The $P$. aeruginosa PAO genetic map carries a proline utilisation marker ( $p r u-365)$ between pru-70 and cys-59 (figure). To determine the relative positions of $m u c-3739$ and pru-365 on the genetic map, PAM739 (pMO60) was plate mated with PAO6021. When proB65 $5^{+}$was the selected marker, $99 \%$ of $\mathrm{Muc}^{+}$recombinants were $\mathrm{Pru}^{+}$. Because $\mathrm{Muc}^{-} \mathrm{Pru}^{+}$recombinants were not recovered, the most probable order of the markers is muc-pru-pro. For reasons that we are unable to explain, $\mathrm{Pru}^{+}$ recombinants were not recovered by direct selection, even though such recombinants can be recovered at the expected frequencies in $\mathrm{PAO} \times \mathrm{PAO}$ crosses.

\section{Discussion}

The transfer of a locus causing the production of alginate in $P$. aeruginosa isolates from CF patients to $P$. aeruginosa PAO, and its subsequent expression in that strain, opens the way to a genetic analysis of the nature of this locus, and will allow a comparison with those $P$. aeruginosa PAO loci that have been identified by Fyfe and Govan (1983) as being responsible for alginate production. These workers have mapped two such loci, closely linked to but on either side of $c y s-59$, and phenotypically distinguishable on the basis of colony morphology. Group-1 mutations, represented by muc-23 which maps to the right of cys-59, yield mucoid colonies on minimal agar, whereas group-2 mutations, represented by $m u c-22$ which maps to the left of cys -59 , gives rise to non-mucoid colonies on minimal agar. All five PAM strains generated in this study form mucoid colonies on minimal agar and hence carry group-1 mutations but our linkage data clearly indicate a position for $m u c$ to the left of $c y s-$ 59. Furthermore, although muc-22 maps to the right of pru (Fyfe and Govan, 1983), our data suggest that muc-3739 (carried by PAM404) maps to the left of pru. Clearly, more than one locus is involved, and more precise mapping of further mutations either generated in P. aeruginosa PAO or transferred to PAO from mucoid clinical isolates is required before any conclusions can be drawn.

It is more difficult to correlate our findings with those of Ohman and Chakrabarty (1981) who investigated three closely linked loci, denoted alg, in a mucoid clinical isolate that could mutate to suppress the mucoid phenotype. Moreover, the location of these alg loci was determined by Ohman and Chakrabarty in relation to a range of auxotrophic markers generated in their clinical isolate. Thus is is not possible to relate the location of any of these loci to the $P$. aeruginosa PAO chromosome which serves as a reference point for genetic studies in this organism. In a later study, and using another mucoid clinical isolate, Darzins and Chakrabarty (1984) mapped a mutation that suppressed the mucoid phenotype to $19^{\prime}$ on the PAO chromosome map. They were able to do this because a clone from a cosmid gene bank, identified by its ability to complement the alg $^{-}$suppressor mutation (i.e., restore the mucoid phenotype), also carried the $\operatorname{argH} H^{+}$wild-type gene which is located at $19^{\prime}$ (Holloway and Matsumoto, 1984). These workers also identified three other $\mathrm{alg}^{-}$loci on the basis of complementation by different clones from their cosmid bank, but were not able to determine the chromosomal locations of these loci. Isolation and mapping of the mucoid suppressor mutations in the PAM strains described in this paper will determine the map positions of suppressor loci.

A reported feature of mucoid strains is their instability during culture (Zierdt and Schmidt, 1964; Govan, 1975; Fyfe and Govan, 1983). Govan (1975) reported that the use of desoxycholate citrate agar allows maintenance of the mucoid colony morphology. We noted early in this study that our strains appeared quite stable for the mucoid phenotype. Boyce and Miller (1981) reported that supplementation of the growth medium with $0.03 \mathrm{M} \mathrm{FeCl}$ led to rapid overgrowth of the culture by nonmucoid revertants, and preliminary studies with our PAM strains support this (data not shown). This leads to interesting speculations as to the role of $\mathrm{Fe}^{+++}$in the emergence and maintenance of the mucoid phenotype in the lungs of CF patients.

This work was supported by a grant from the National Health and Medical Research Council (NHMRC) of Australia. We thank Beecham (Australia) for a gift of carbenicillin. 


\section{REFERENCES}

Bachmann B J 1983 Linkage map of Escherichia coli K-12, Edition 7. Microbiological Reviews 47:180-230.

Bagdasarian M, Bagdasarian M M, Coleman S, Timmis K N 1979 New vector plasmids for cloning in Pseudomonas. In: Timmis K N, Puhler A (eds) Plasmids of medical, environmental and commercial importance. Elsevier/North Holland, Amsterdam. pp. 411-422.

Banerjee P C, Vanags R I, Chakrabarty A M, Maitra P K 1983 Alginic acid synthesis in Pseudomonas aeruginosa mutants defective in carbohydrate metabolism. Journal of Bacterio$\log y$ 155:238-245.

Boyce J R, Miller R V 1982 Selection of non-mucoid derivatives of mucoid Pseudomonas aeruginosa is strongly influenced by level of iron in the culture medium. Infection and Immunity 37:695-701.

Darzins A, Chakrabarty A M 1984 Cloning of genes controlling alginate biosynthesis from a mucoid cystic fibrosis isolate of Pseudomonas aeruginosa. Journal of Bacteriology 159:9-18.

Dean H F, Royle P, Morgan A F 1979 Detection of FP-plasmids in hospital isolates of Pseudomonas aeruginosa. Journal of Bacteriology 138:249-250.

Doggett R G, Harrison G M, Stillwell R N, Wallis E S 1966 An atypical Pseudomonas aeruginosa associated with cystic fibrosis of the pancreas. Journal of Pediatrics 68:215-221.

Fyfe J A M, Govan J R W 1980 Alginate synthesis in mucoid Pseudomonas aeruginosa: a chromosomal locus involved in control. Journal of General Microbiology 119:443-450.

Fyfe J A M, Govan J R W 1983 Synthesis, regulation and biological function of bacterial alginate. Progress in Industrial Microbiology 18:46-83.

Gillies R R, Govan J R W 1966 Typing of Pseudomonas pyocyanea by pyocine production. Journal of Pathology and Bacteriology 91:339-345.

Govan J R W 1975 Mucoid strains of Pseudomonas aeruginosa: the influence of culture medium on the stability of mucus production. Journal of Medical Microbiology 8:513-522.

Govan J R W, Fyfe J A M 1978 Mucoid Pseudomonas aeruginosa and cystic fibrosis: resistance of the mucoid form to carbenicillin, flucloxacillin and tobramycin and isolation of mucoid variants in vitro. Journal of Antimicrobial Chemotherapy 4:233-240.

Haas D, Holloway B W 1976 R factor variants with enhanced sex factor activity in Pseudomonas aeruginosa. Molecular and General Genetics 144:243-251.

Henry R L, Dorman D C, Brown J, Mellis C 1982 Mucoid Pseudomonas aeruginosa in cystic fibrosis. Australian Paediatric Journal 18:43-45.
Hodson M E, Penketh A R L, Batten J C 1981 Aerosol carbenicillin and gentamicin treatment of Pseudomonas aeruginosa infection in patients with cystic fibrosis. Lancet 2:1137-1139.

Holloway B W 1965 Variations in restriction and modification of bacteriophage following increase of growth temperature of Pseudomonas aeruginosa. Virology 25:634-642.

Holloway B W, Krishnapillai V, Morgan A F 1979 Chromosomal genetics of Pseudomonas. Microbiological Reviews 43:73-102.

Holloway B W, Matsumoto H 1984 Pseudomonas aeruginosa. In: O'Brien S (ed). Genetic maps, vol 3. Cold Spring Harbor, New York. pp 194-197.

Leisinger T, Haas D, Hegarty M P 1972 Indospicine as an arginine antagonist in Escherichia coli and Pseudomonas aeruginosa. Biochimica et Biophysica Acta 262:214-219.

May J R, Herrick N C, Thompson D 1972 Bacterial infection in cystic fibrosis. Archives of Diseases of Childhood 47:908-913.

Ohman D E, Chakrabarty A M 1981 Genetic mapping of chromosomal determinants for the production of the exopolysaccharide alginate in a Pseudomonas aeruginosa cystic fibrosis isolate. Infection and Immunity 33:142-148.

Pugashetti B K, Vadas L, Prihar H S, Feingold D S 1983 GDP mannose dehydrogenase and biosynthesis of alginate-like polysaccharide in a mucoid strain of Pseudomonas aeruginosa. Journal of Bacteriology 153:1107-1110.

Riess G, Holloway B W, Puhler A 1980 R68.45, a plasmid with chromosome mobilising ability (Cma) carries a tandem duplication. Gentical Research 36:105-115.

Royle P L, Matsumoto H, Holloway B W 1981 Genetic circularity of the Pseudomonas aeruginosa PAO chromosome. Journal of Bacteriology 145:145-155.

Soldati L, Crockett R, Carrigan J M, Leisinger T, Holloway B W, Haas D 1984 Revised locations of the hisI and pru (proline utilization) genes on the Pseudomonas aeruginosa chromosome map. Molecular and General Genetics 193:431436.

Stanisich V A, Holloway B W 1972 A mutant sex factor of Pseudomonas aeruginosa. Genetical Research 19:91-108.

Watson J M, Holloway B W 1976 Suppressor mutations in Pseudomonas aeruginosa. Journal of Bacteriology 125:780786.

Wood R E, Boat T F, Doershuk C F 1976 State of the art-cystic fibrosis. American Review of Respiratory Diseases 113:833878.

Zierdt C H, Schmidt P J 1964 Dissociation in Pseudomonas aeruginosa. Journal of Bacteriology 87:1003-1010. 